\title{
Ophthalmic biosimilars and biologics-role of endotoxins
}

\author{
Ashish Sharma $^{1}$ - Nilesh Kumar $\mathbb{D}^{1} \cdot$ Baruch D. Kuppermann ${ }^{2} \cdot$ Francesco Bandello $^{3}$ - Anat Loewenstein ${ }^{4}$
}

Received: 13 September 2019 / Accepted: 26 September 2019 / Published online: 16 October 2019

(c) The Royal College of Ophthalmologists 2019

The sterile endophthalmitis has been reported in all anti vascular endothelial growth factor (VEGF) formulations used for intravitreal therapy such as bevacizumab, ranibizumab, aflibercept, and pegaptanib, with varying incidence rate [1]. Infectious endophthalmitis is the most dreaded complication of this therapy, with an incidence of $0.029-0.058 \%$ [2]. The cumulative rate increases up to $0.843 \%$ per person when 60 intra-vitreal treatments (IVT) were administered. The rate of non-infectious or sterile endophthalmitis increased up to $0.228 \%$ per person when 20 IVTs were administered and remained stable till 60 IVTs [3].

Previously, sterile inflammation has been reported in patients receiving razumab (Intas Pharmaceuticals Ltd, Ahmedabad, India), the first biosimilar of ranibizumab approved for ophthalmic use in India. These reports led to a recall of the drug by the manufacturer and subsequent changes in the formulation [4]. Abicipar pegol, an innovative designed ankyrin repeat proteins (DARPin) molecule under evaluation, has been modified due to the high rate of intra-ocular inflammation (IOI) in clinical trials. The MAPLE trial for the drug has reported a rate of IOI at $8.9 \%$, with $1.6 \%$ severe reaction [5]. The mechanism of the sterile endophthalmitis in anti-VEGF therapy has not been clearly understood. The cases include patients invoking immune reaction to the biological drug itself, an entity known as anti-drug antibody; or reaction to the impurities present in

$\triangle$ Ashish Sharma

drashish79@hotmail.com

1 Department of Vitreoretina, Lotus Eye Hospital and Institute, Coimbatore, Tamil Nadu, India

2 Gavin Herbert Eye Institute, University of California, Irvine, Irvine, CA, USA

3 Department of Ophthalmology, University Vita-Salute, Scientific Institute San Raffaele, Milano, Italy

4 Division of Ophthalmology, Tel Aviv Sourasky Medical Center and Sackler Faculty of Medicine, Tel Aviv University, Tel Aviv, Israel the drug formulation [6]. There have been several reports of cluster sterile endophthalmitis arising from intravitreal bevacizumab therapy [7-9]. Wang et al. reported cluster of sterile endophthalmitis $(69 \%)$ due to counterfeit bevacizumab. On analysis of the counterfeit vial, it was found to be having $32 \mathrm{EU} / \mathrm{ml}$ of endotoxin, and endotoxin was demonstrated in the vitreous samples [7]. The FDA recommends an EL of $0.2 \mathrm{EU} / \mathrm{ml}$ for the pharmaceuticals for intraocular use [1, 10].

Endotoxins are the lipopolysaccharide complexes found in the outer cell wall of gram-negative bacteria. These endotoxins found in ophthalmic pharmaceuticals have two sources of induction, namely intrinsic and extrinsic. Intrinsic endotoxins are found to be originating from the inherent manufacturing process of the drug. Extrinsic endotoxins are introduced by improper sterilization and storage processes. These endotoxins have been proven to cause toxic anterior segment syndrome (TASS), a wellestablished non-infectious anterior uveitis entity following exposure to intraocular pharmaceuticals during ocular surgeries [11]. International Standards Organization (ISO) had recommended the EL to be below $0.5 \mathrm{EU} / \mathrm{ml}$, which FDA thought not to be effective in preventing TASS and has now revised the EL to $0.2 \mathrm{EU} / \mathrm{ml}[1,11]$. Razumab followed ISO recommendation regarding EL. However, it further modified it as per FDA recommendation after few reports of inflammation. (Unpublished data from manufacturers).

Endotoxin-induced uveitis (EIU) is the sterile inflammation of the uveal tissue of the eye following an exposure to lipopolysaccharides of the Gram-negative bacterial cell wall. These endotoxins are intrinsic in origin as the biologics involve cell cultures to produce the molecule. Biologics such as ranibizumab and aflibercept are produced from mammalian cell lines, whereas new research molecules such as DARPins are produced from bacterial cells (E. coli) and thus may have a higher propensity to have intrinsic endotoxins. This could be a possible cause of the intra-ocular inflammation (IOI) in clinical trial results of abicipar. Phase 3 trials of abicipar showed an IOI rate of $\sim 16 \%$. Evaluation of the formulation showed $E$. coli particles as impurities, 
which led to the refinement of the formulation utilized in the subsequent MAPLE trial that resulted in a reduction of IOI of $\sim 7.1 \%$ [5]. According to the recent data shared at the American Academy of Ophthalmology (AAO) 2019 annual meeting, the inflammation rate has come down to $1.9 \%$. The processes to detoxify drugs produced from $E$. coli cell lines to reduce the endotoxin load themselves have a scope of significant improvement [12]. Brolucizumab, an scFv molecule that has received FDA approval, is also produced from microbial cell lines [13]. The phase 3 clinical trial of brolucizumab has shown an uveitis rate of $\sim 2.2 \%$ [14].

The FDA recommends an EL for ophthalmic pharmaceuticals, solutions, and devices which are being used in anterior segment surgery, such as intra-ocular lens, ophthalmic visco-surgical devices, and balanced salt solutions. EIU in rabbit model has shown that vitreal cavity is $\sim 2-$ to 10 -fold more sensitive to endotoxin administration than the anterior chamber, and thus potentially requires a lower recommended EL $[15,16]$.

To the best of our knowledge, EL limits for intravitreal therapy have not been specified by the FDA, which would appear to be a deficiency. In the era of intravitreal injection and biosimilars on the horizon, it would be helpful if such recommendations were established to make these therapies safer by reducing the risk of inflammation.

\section{Compliance with ethical standards}

Conflict of interest AS conflicts of interest: CONSULTANT: Novartis India, Allergan Global, Intas India, Bayer India. NK: None. BDK conflicts of interest: CLINICAL RESEARCH: Alcon, Alimera, Allegro, Allergan, Apellis, Clearside, Genentech, GSK, Ionis, jCyte, Novartis, Regeneron, ThromboGenics; CONSULTANT: Alimera, Allegro, Allergan, Cell Care, Dose, Eyedaptic, Galimedix, Genentech, Glaukos, Interface Biologics, jCyte, Novartis, Ophthotech, Regeneron, Revana, Theravance Biopharma. Francesco Bandello conflicts of interest: CONSULTANT: Allergan, Bayer, Boehringer- Ingelheim, Fidia Sooft, Hofmann La Roche, Novartis, NTC Pharma, Sifi, Thrombogenics, Zeiss. AL conflicts of interest: CONSULTANT: Allergan, Novartis, Roche, Notal Vision, Fiorsightslabs, Beyeonics, Bayer Health Care.

Publisher's note Springer Nature remains neutral with regard to jurisdictional claims in published maps and institutional affiliations.

\section{References}

1. Agrawal S, Joshi M, Christoforidis JB. Vitreous Inflammation associated with intravitreal anti-VEGF pharmacotherapy. Mediators Inflamm. 2013;2013:1-6.
2. Sigford D, Reddy S, Mollineaux C, Schaal S. Global reported endophthalmitis risk following intravitreal injections of antiVEGF: a literature review and analysis. Clin Ophthalmol. 2015;9:773-81.

3. Daien V, Nguyen V, Essex RW, Morlet N, Barthelmes D, Gillies $\mathrm{MC}$, et al. Incidence and outcomes of infectious and noninfectious endophthalmitis after intravitreal injections for age-related macular degeneration. Ophthalmology. 2018;125:66-74.

4. Sharma A, Kumar N, Kuppermann B, Francesco B, Lowenstein A. Ophthalmic biosimilars: lessons from India. Indian J Ophthalmol. 2019;67:1384.

5. Allergan and molecular partners announce topline safety results from MAPLE study of abicipar pegol-molecular partners. https://www.molecularpartners.com/allergan-and-molecular-pa rtners-announce-topline-safety-results-from-maple-study-of-a bicipar-pegol/.

6. Sharma A, Kumar N, Kuppermann BD, Francesco B, Loewenstein A. Biotherapeutics and immunogenicity: ophthalmic perspective. Eye. 2019. http://www.nature.com/articles/s41433-0190434-y.

7. Wang F, Yu S, Liu K, Chen F-E, Song Z, Zhang X, et al. Acute intraocular inflammation caused by endotoxin after intravitreal injection of counterfeit bevacizumab in Shanghai, China. Ophthalmology. 2013;120:355-61.

8. Ricci F. A cluster of noninfectious endophthalmitis after intravitreal bevacizumab: long-term follow-up. DJO. 2016 http://djo.ha rvard.edu/site.php?url=/physicians/oa/2733.

9. Sinha S, Vashisht N, Venkatesh P, Garg S. Managing bevacizumab-induced intraocular inflammation. Indian $\mathrm{J}$ Ophthalmol. 2012;60:311.

10. Endotoxin testing recommendations for single-use intraocular ophthalmic devices. guidance for industry and food and drug administration staff. U.S. Food and Drug Administration (CDRH, Office of Device Evaluation Division of Ophthalmic and Ear, Nose, and Throat Devices.) 2015. http://www.fda.gov/downloads/ medicaldevices/deviceregulationandguidance/guidancedocuments/ ucm393376.pdf. FDA-2014-D-0332. (Accessed 7 Sep 2019).

11. Vijayakumar R, Aboody MSA, Alturaiki W, Sandle T. Review on endotoxin mediated toxic anterior segment syndrome (TASS) in ophthalmic products-outbreaks, product recall and testing limits. EJPPS - Phss. 2016;22:1-6.

12. Petsch D. Endotoxin removal from protein solutions. J Biotechnol. 2000;76:97-119.

13. Bates A, Power CA. David vs. Goliath: the structure, function, and clinical prospects of antibody fragments. Antibodies 2019;8:28.

14. Dugel PU, Koh A, Ogura Y, Jaffe GJ, Schmidt-Erfurth U, Brown DM, et al. HAWK and HARRIER: Phase 3, multicenter, randomized, double-masked trials of brolucizumab for neovascular age-related macular degeneration. Ophthalmology. 2019; S0161642018330185.

15. Bantseev V, Miller PE, Bentley E, Schuetz C, Streit TM, Christian $\mathrm{BJ}$, et al. Determination of a no-observable effect level for endotoxin following a single intravitreal administration to Dutch belted rabbits. Invest Ophthalmol Vis Sci 2017;58:1545.

16. Bantseev V, Miller PE, Nork TM, Rasmussen CA, McKenzie A, Christian BJ, et al. Determination of a no observable effect level for endotoxin following a single intravitreal administration to cynomolgus monkeys. J Ocul Pharmacol Therapeutics. 2019;35:245-53. 